\title{
Figuring of optical aluminium devices by reactive ion beam etching
}

\author{
Jens Bauer ${ }^{1, *}$, Melanie Ulitschka ${ }^{1}$, Frank Frost $^{1}$, and Thomas Arnold ${ }^{1,2}$ \\ ${ }^{1}$ Leibniz Institute of Surface Engineering (IOM), Permoserstrasse 15, D-04318 Leipzig, Germany \\ ${ }^{2}$ TU Dresden, Institute of Manufacturing Science and Engineering, George-Bähr-Strasse 3c, D-01062 Dresden, Germany
}

\begin{abstract}
Ion beam figuring (IBF) is an established method in high-end surface manufacturing. However, the direct processing of desired materials as standard Al alloys (e.g. Al6061) fails, since the surface roughness increases drastically as a result of inhomogeneous etching due to structural, crystallographic and chemical irregularities inside the material matrix. As an alternative figuring technology reactive ion-beam etching (RIBE) is a promising route. RIBE provides the direct machining of $\mathrm{Al}$ alloys while preserving the surface roughness almost in its initial state. The RIBE process with nitrogen gas is focused more detailed in this study.
\end{abstract}

\section{Introduction}

Metal optics are of increasing interest, since refractive optical elements reach their physical limitations within the technological progress in EUV/XUV lithography, $\mathrm{x}$-ray and synchrotron optics. The upcoming needs of most flexible shape adjustment and increasing surface quality demand improved deterministic tools in ultra-precision surface machining. RIBE is a promissing technique for figure error correction of $\mathrm{Al}$ mirror devices. In addition to conventional IBF the process gas contains chemically active species as oxygen [1] or nitrogen [2] changing the etch mechanism from pure sputtering to a chemical-assisted sputter etch process. The direct ion-beam machining process has been optimized for figure error correction of standard Al alloy surfaces with focus on superior optical surface quality.

\section{Experimental}

Planar single-point diamond turned (SPDT) Al samples (supplied from IOF Jena) were made from the RSA alloys Al6061 and A1905 (RSP technology).

For figure error correction low-energy, narrow ion beams $(\leq 1.5 \mathrm{keV})$ are used in deterministic operation via a dwell-time approach. Aperture-free ion beams with a Gaussian shape and a full-width at half maximum (FWHM) of about $5-7 \mathrm{~mm}$ and sub-aperture ion beam tools with FWHM $\geq 0.6 \mathrm{~mm}$ are available [3]. Latter allows figure error correction from the low-spatial frequency to the mid-spatial frequency range $<0.5 \mathrm{~mm}^{-1}$. By use of a six-axes CNC motion setup for sample movement, also deep and arbitrarily shaped devices can be corrected [4].

The surface morphology is analyzed by optical microscopy, white light interferometry (WLI), and atomic force microscopy (AFM). Power spectral density (PSD) functions are calculated for roughness analysis.
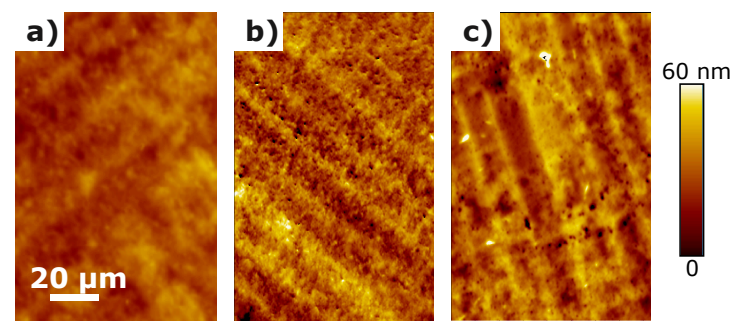

Figure 1. Surface topographies by WLI: a) an untreated Al905 surface after SPDT with a rms roughness of $R_{q}=10.9 \mathrm{~nm}, b$ ) argon ion treatment $(1.5 \mathrm{keV}$, etching depth: $330 \mathrm{~nm})$ results in considerable roughening with $\mathrm{R}_{\mathrm{q}}=21.0 \mathrm{~nm}$, and c) nitrogen ion treatment $(1.5 \mathrm{keV}$, etching depth: $1 \mu \mathrm{m})$ with an almost preserved initial topography at $\mathrm{R}_{\mathrm{q}}=12.2 \mathrm{~nm}$.

\section{Results and discussion}

RIBE processing with oxygen and nitrogen as reactive gas species allows the direct figuring of $\mathrm{Al}$ surfaces [1, 2]. In contrast to the classical RIBE scheme, no volatile process products are generated, but the machined surface is determinately chemically modified. In particular, an in situ forming surface layer acts as an etch front moderating the inhomogeneous structural conditions of the Al matrix. Etching occurs as physical sputter erosion within this surface layer exclusively. A very stable and non-hazardous etch mechanism is obtained. As a remarkable technological achievement, the surface roughness is preserved almost in its initial state during RIBE processing while conventional IBF with argon gas results in a considerable surface roughening (Fig. 1). Thus, the RIBE technique permits ultra-precision figure error correction of high-quality optical Al surfaces.

The RIBE process conditions have been investigated in more detail with a special focus on the preservation of the initial topography (Fig. 2). The ion implantation depth depends on the ion energy. Thus, an effect on the ni-

*e-mail: jens.bauer@iom-leipzig.de 

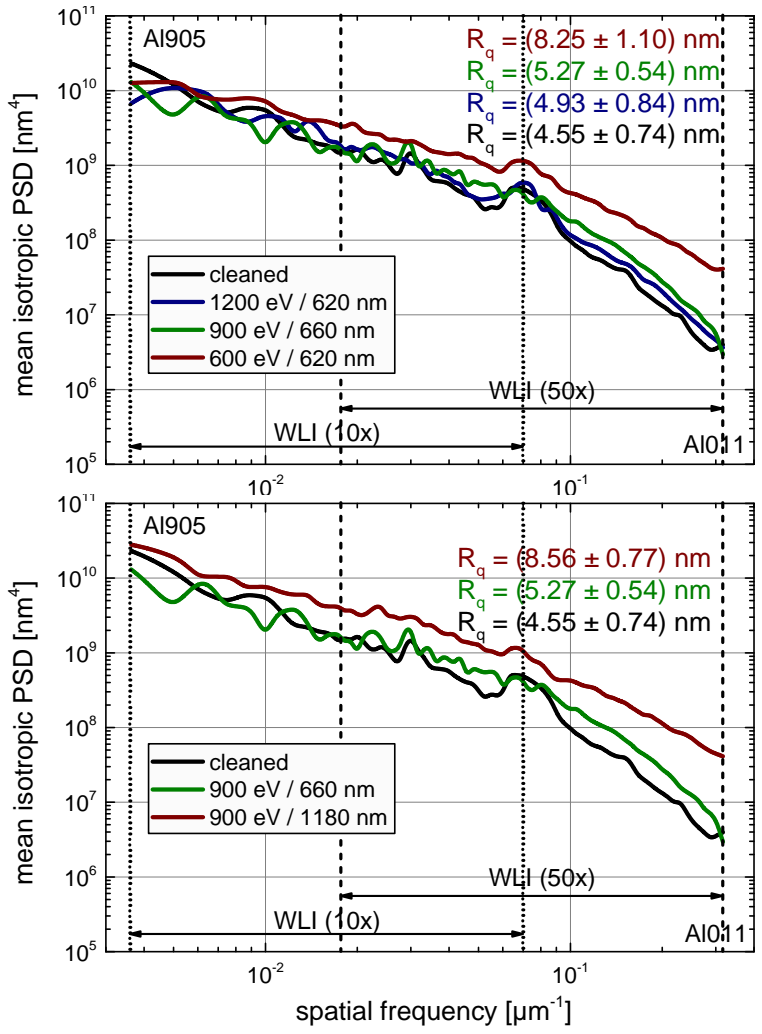

Figure 2. PSD functions before and after RIBE processing with nitrogen gas. Surface degradation increases at lower ion energies (upper) and for increased process time and thus depth (lower).
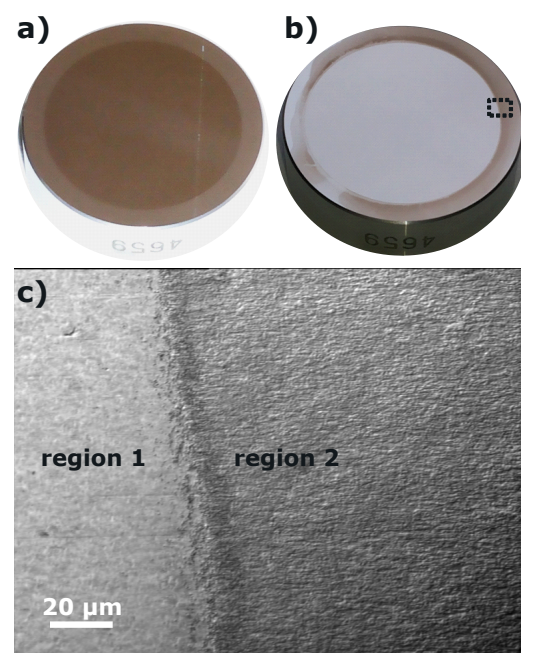

Figure 3. a) Aluminium sample after nitrogen ion beam treatment $(1.5 \mathrm{keV})$ applying a graphite contact mask with $38 \mathrm{~mm}$ opening. b) The nitride layer was removed by wet-chemical processing with a $1 \% \mathrm{KOH}$ solution. c) Microscopy image of the marked area in b). Region 1 defines the sample area, which was first ion beam treated and subsequently wet-chemically etched. Region 2 indicates the area, which was masked during ion beam treatment and thus only wet-chemically etched. tride layer formation can be expected. At low ion energies $<900 \mathrm{eV}$ some surface degradation was observed already at $\sim 600 \mathrm{~nm}$ etch depth. For ion energies $>900 \mathrm{eV}$ the initial topography is almost preserved up to $1 \mu \mathrm{m}$ etch depth.

However, the surface nitride lowers the reflection properties of the Al surface. Thus, the nitride removal by wetchemical treatment has been focused. AlN is highly resistant against most chemical etchants including $\mathrm{HCl}$. Considerable AlN etching performance is provided only by $\mathrm{KOH}-$ based etch solutions and to minor extent by $\mathrm{NaOH}-$ based etchants. A sequence of treatment in $1 \%$ aqueous $\mathrm{KOH}$ solution and subsequent rinsing in DI-water has been applied (Fig. 3a/b). The etching rate is $4.4 \AA / \mathrm{s}$. In Fig. 3c the border area of nitride layer (region 1) and clean aluminium surface (region 2) is shown after wet-chemical etching. Since the etch stop has been chosen very accurately to the nitride layer thickness the initially nitrided region 1 exhibits a very smooth surface free of any nitride residuals. In contrast, the clean aluminium degraded considerably as a result of strong etching. Hence, a precise etch stopping is necessary for successful nitride removal.

In summary, for direct surface figuring of optical $\mathrm{Al}$ surfaces the RIBE process conditions with nitrogen gas are addressed. At ion energies $>900 \mathrm{eV}$ the topography is preserved up to $1 \mu \mathrm{m}$ etch depth. With decreasing ion energy and increasing process time surface degradation issues need to be accepted. However, the formation of a homogeneous surface nitride layer is observed for any process condition. Since the optical surface properties are diminished by the nitride, a wet-chemical etching approach for the removal of the nitride layer is successfully applied. T. Liebeskind is thanked for wet-chemical sample treatment.

Financial support given by the German Federal Ministry of Education and Research (BMBF) within InnoProfile Transfer 03IPT706X is gratefully acknowledged.

\section{References}

[1] J. Bauer, F. Frost, T. Arnold, J. Phys. D. Appl. Phys. 50, 085101 (2017)

[2] J. Bauer, M. Ulitschka, F. Frost, T. Arnold, Figure error correction of aluminium mirrors by deterministic reactive ion-beam machining, in EOS Opt. Technol. / 5th Conf. Manuf. Opt. Syst. (European Optical Society, Munich / Germany, 2017)

[3] J. Bauer, M. Ulitschka, F. Frost, T. Arnold, L. Alber, M. Sondermann, G. Leuchs, Ultra-precision surface figuring of optical aluminium devices, in OSA Adv. Photonics Congr. / Nov. Opt. Mater. Appl. Top. Meet. (Optical Society of America, Zurich / Switzerland, 2018)

[4] J. Bauer, M. Ulitschka, F. Pietag, T. Arnold, J. Astron. Telesc. Instrum. Syst. 4, 046003 (2018) 